\title{
Full polarization VLBA maps of a proto-planetary nebula
}

\author{
Kim McAlpine ${ }^{1}$, A. J. Kemball ${ }^{2}$ \\ and J. L. Jonas ${ }^{1}$ \\ ${ }^{1}$ Department of Physics and Electronics, Rhodes University, Grahamstown, 6139, South Africa \\ ${ }^{2}$ National Center for Supercomputing Applications, University of Illinois at \\ Urbana-Champaign, 605 East Springfield Avenue, Champaign, IL 61820, USA
}

\begin{abstract}
The morphological evolution of stars from the asymptotic giant branch (AGB) to the planetary nebula $(\mathrm{PN})$ phases presents an intriguing problem in stellar research. Planetary nebulae show bright-rims, well-defined shell-like structures and a large proportion of them are axisymmetric. In contrast their progenitor AGB stars are largely spherically symmetric (Sahai, 2004). Studies of proto-planetary nebulae, which are objects in transition between these two phases of stellar evolution, offer insight into the mechanisms which are responsible for the onset of axisymmetry. This poster presents the first polarization VLBI observations of the $1612 \mathrm{MHz}$ $\mathrm{OH}$ maser emission from the proto-planetary nebula candidate $\mathrm{OH} \mathrm{O} .9+1.3$. The morphology, kinematics and polarization properties of these masers are discussed.
\end{abstract}

Keywords. proto-planetary nebulae, $\mathrm{OH}$ masers

\section{Introduction}

The period immediately following the asymptotic giant branch (AGB) is one of the least understood phases of stellar evolution and there remain many open questions regarding the transformation of AGB stars into planetary nebulae. Many promising models detailing the morphological evolution of late-type stars invoke the role of stellar magnetic fields (e.g. Matt et al. 2004; García-Segura et al. 2005). Maser polarization measurements can be of considerable use in establishing the morphology and magnitude of such fields. Thus the polarization VLBI maps of $\mathrm{OH} 0.9+1.3$ presented in this work were used to investigate the morphology and kinematics of this source with a view to gaining insight into the relative dynamic influence of magnetic fields and the development of asymmetry in post-AGB objects.

\section{Observations}

The source was observed with the VLBA on the 24th and 25th of October 1994. The total observing run lasted 12 hours and included interleaved observations of continuum calibrators. The observations were done in spectral line mode and the data was recorded in dual circular polarization in two overlapping spectral windows of $250 \mathrm{kHz}$ each.

\section{Results}

The spectrum of $\mathrm{OH} 0.9+1.3$ consists of several strong, $\sim 50 \mathrm{Jy}$, blue-shifted peaks at $\sim-124 \mathrm{~km} \cdot \mathrm{s}^{-1}$ and a considerably weaker red-shifted emission feature of $600 \mathrm{mJy}$ at $-93 \mathrm{~km} \cdot \mathrm{s}^{-1}$. Contour maps of the Stokes I emission of both the blue- and red-shifted emission from this source are presented in Figure 1. 


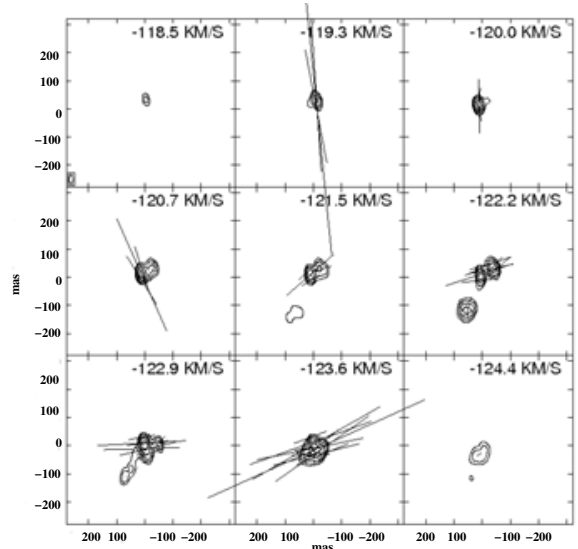

(a)

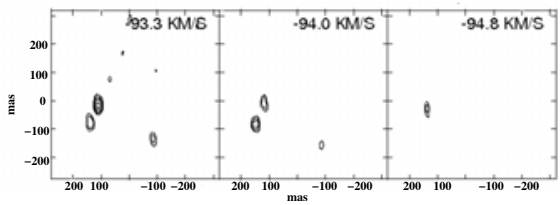

(c)

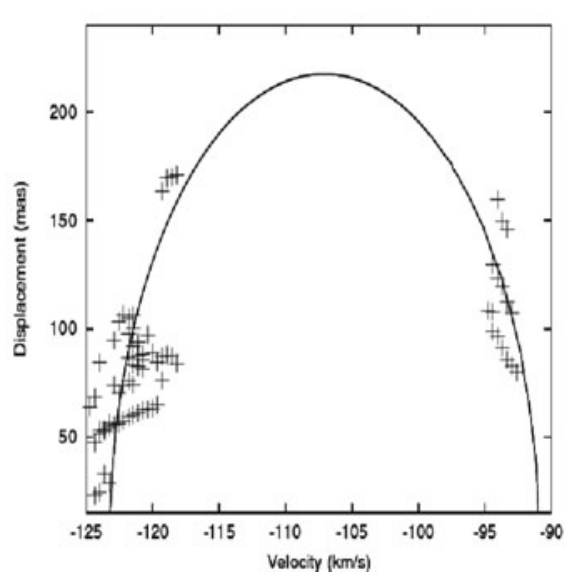

(b)

Figure 1. a) Contour maps of blue-shifted emission from $\mathrm{OH} 0.9+1.3$. Contours are plotted at $0.368 \times(-1,1,2,4,8,16,32) \mathrm{Jy} /$ Beam. The magnitude and angle of the linear polarization vectors are shown by the length and direction of the vectors; here 1 mas $=2.00 \mathrm{mJy} /$ Beam of polarized emission. b) An expanding shell model fit to the maser components. The solid line indicates the model and the crosses represent the displacement of the maser components relative to the assumed stellar position. c) Contour maps of red-shifted emission from $\mathrm{OH} \mathrm{O} .9+1.3$. Contours are plotted at $19.3 \times(-1,1,2,4,8,16,32) \mathrm{mJy} /$ Beam.

\section{Conclusion}

The total intensity maser maps demonstrated a considerable degree of asymmetry with the blue- and red-shifted emission located in spatially distinct regions of the envelope. The morphology of this source is not consistent with the standard symmetric thin-shell model and an attempt to fit the traditional $\mathrm{OH} / \mathrm{IR}$ kinematic model of a simple expanding shell to the maser components was found to be unsatisfactory. The fractional linear and circular polarizations of the maser components were derived from the Stokes parameter maps. In all except one of the components the total fractional polarization was found to be low $(<15 \%)$. The mean fractional linear and circular polarization were calculated to be $5.5 \%$ and $7.1 \%$ respectively. The absence of an identifiable Zeeman pair in the Stokes $\mathrm{V}$ map prohibited the estimation of the magnetic field in this source.

\section{Acknowledgements}

I would like to acknowledge the financial assistance of Rhodes University, in the form of a Henderson award, and SKA South Africa during the course of the research.

\section{References}

Sahai, R. 2004, ASP CS 313, 141

García-Segura, G., López, J. A. \& Franco, J. 2005 ApJ 618, 919

Matt, S., Frank, A. \& Blackman, E. G. 2004 ASP CS 313, 449 